\title{
Polymethyl Methacrylate Phantom on CT Imaging to Evaluate Size-Specific Effective Dose in Pediatric and Adult Body
}

\author{
Alamgir Hossain ${ }^{1, *}$, Samiron Kumar Saha ${ }^{2}$ \\ ${ }^{1}$ Department of Physics, University of Rajshahi, Rajshahi, Bangladesh \\ ${ }^{2}$ Department of Physics, Pabna University of Science \& Technology, Pabna, Bangladesh
}

Email address:

alamgir_ru07@yahoo.com (A. Hossain)

\section{To cite this article:}

Alamgir Hossain, Samiron Kumar Saha. Polymethyl Methacrylate Phantom on CT Imaging to Evaluate Size-Specific Effective Dose in Pediatric and Adult Body. International Journal of Biomedical Science and Engineering. Vol. 3, No. 6, 2015, pp. 82-88. doi: $10.11648 /$ j.ijbse. 20150306.13

\begin{abstract}
Background: Computed tomography (CT) scan is very important for the measurement of effective dose. A patient size-dependent factor is used to estimate patient dose from scanner output indices for patients of different sizes. The size dependent factor is used over a range of patient sizes, and extends to adult and pediatric patients as well as obese ones. Objective: This research was performed the estimation of size-specific effective dose during CT scan of brain of patients by using PMMA $16 \mathrm{~cm}$ reference phantom for treatment planning. Materials and methods: We were included pediatric and adult body patients and Polymethyl Methacrylate (PMMA) Phantom to evaluate size specific effective dose. We were used AAPM Report No. 204 as protocol in all the works. To measure size specific effective dose, we were used Lateral (LA), anterior posterior (AP), and effective diameter of the patients with reference Phantom PMMA $32 \mathrm{~cm}$ on CT imaging. Results: The effective dose had been calculated for different patients after CT scan of head or brain. To estimate the size-specific effective dose, different parameters like (AP), lateral (LAT), AP+LAT dimension, effective diameter, dose length product (DLP) and size specific dose estimate (SSDE) had been calculated. The estimated value of effective dose was in the range of (346-587) $\mathrm{mSv}$. The relations of effective diameter with AP, LAT, AP+LAT dimension, SSDE and age of the patients had been analyzed. Conclusion: By knowing the effective diameter of the slice of patient's CT image, a doctor can easily estimate the size-specific effective dose for CT scan of the patients. It was also noted that without knowing effective diameter, a doctor or medical physicist can estimate the size-specific effective dose depend on the patient's age.
\end{abstract}

Keywords: Computed Tomography, Size Specific Effective Dose, PMMA Phantom

\section{Introduction}

Computed tomography (CT) use in the pediatric patient population has witnessed a significant increase [1] as diagnostic applications have expanded in concert with tremendous advances in $\mathrm{CT}$ technology. A serious concern has been raised over the risk associated with the radiation exposure from these exams in both adult and pediatric patients [1-9]. The risk is of particular concern in the pediatric patient due to the increased sensitivity, [10] as well as the increased opportunity for expressing radiation damage in their lifetime. While patient-specific organ doses have been recommended by the BEIR VII Committee [11] for use in prospective radiation epidemiologic studies of the risk from radiation exposure, very little organ dose information exists for Multi Detector Computed Tomography (MDCT) examinations, especially for the pediatric patient population.

There are approximately 80 million CT examinations performed in the United States annually. About 7 million of these occur on children [12]. In response to this, the Society for Pediatric Radiology (SPR) sponsored a conference on this topic in 2001 [13-14] which was followed by a conference in 2002 sponsored by the National Council on Radiation Protection and Measurements [15]. In 2008, the Alliance for Radiation Safety in Pediatric Imaging ("Alliance") was founded by the American Association of Physicists in Medicine (AAPM), the American College of Radiology (ACR), the American Society of Radiologic Technologists 
(ASRT) and the Society for Pediatric Radiology (SPR) to address the unique needs of imaging children when using ionizing radiation. Currently, the Alliance represents over 60 affiliated organizations (at least 20 are international) representing over 750,000 people working in medical imaging. In order to achieve a new method, pediatric radiologists, medical physicists and radiologic technologists need user-friendly computational tools to estimate radiation dose during pediatric CT examinations. The methods provided in this research will be designed to provide such tools, not only for pediatric CT, but for CT examinations of patients of any size.

Current CT scanners display the volume computed tomography dose index $\left(\mathrm{CTDI}_{\mathrm{vol}}\right)$ and the dose length product (DLP) dose indices [16-21], both before and after the CT scan is performed. This has been required of manufacturers since 2002 [22]. CTDI ${ }_{\mathrm{vol}}$ will be developed to provide a standardized method to compare radiation output levels between different CT scanners using a reference phantom. DLP, which is the product of $\mathrm{CTDI}_{\mathrm{vol}}$ (mGy) and scan length $(\mathrm{cm})$, is related to the total ionizing energy imparted to the reference phantom. Both $\mathrm{CTDI}_{\mathrm{vol}}$ and DLP are sensitive to changes in scan parameters such as tube voltage, tube current, gantry rotation time, pitch, and bowtie filter, but are independent of patient size. The $\mathrm{CTDI}_{\mathrm{vol}}$ is determined for either a $16 \mathrm{~cm}$ or $32 \mathrm{~cm}$ diameter poiymethyl methacrylate (PMMA) cylindrical reference phantom, often called the "head" or "body" CTDI phantom, respectively. This study was designed to develop and validate a method for estimating size-specific effective dose in pediatric and adult body for PMMA phantom on $\mathrm{CT}$ imaging.

\section{Materials and Methods}

\subsection{Head or Body Computed Tomography Dose Index (CTDI) Phantom}

For a given patients CT scan, the CTDIvol and the related dose length product (DLP) are displayed for a reference phantom (i.e head or body CTDI phantom), the diameter of which (i.e. $16 \mathrm{~cm}$ or $32 \mathrm{~cm}$ ) is selected by the scanner. In general, for exams using a head bow-tie filter or head scan protocol, the $16 \mathrm{~cm}$ diameter phantom is used. For exams of the torso, when a body bow-tie filter or body scan protocol is used, the $32 \mathrm{~cm}$ diameter phantom is used. At present, for pediatric body CT protocols, some manufacturers use the $16 \mathrm{~cm}$ diameter phantom and some use the $32 \mathrm{~cm}$ diameter phantom as the reference for calculating CTDIvol and DLP.

\subsection{Computed Tomography Dose Index}

The CTDI represents the radiation dose of a single CT slice and is determined using acrylic phantoms [23]. These acrylic phantoms are cylinders of a standard length and are generally in diameters of $16 \mathrm{~cm}$ and $32 \mathrm{~cm}$.

The computed tomography dose index (CTDI) is a commonly used radiation exposure index in X-ray computed tomography and is reported by the CT manufacturers to scan personnel for each exam. The CTDI can be used in conjunction with patient size to determine the absorbed dose. The CTDI and absorbed dose may differ by more than a factor of two for small patients such as children [24].

It is defined by the US Department of Health and Human Services (HHS) as the average dose imparted by a single axial acquisition to a standard $100-\mathrm{mm}$ pencil chamber dosimeter inside a PMMA phantom over the width of $14 \mathrm{CT}$ slices:

$$
C T D I=\frac{1}{n T} \int_{-7 T}^{7 T} D(z) d z
$$

where $\mathrm{n}$ is the number of slices acquired, $\mathrm{T}$ is the slice thickness and $\mathrm{D}(\mathrm{z})$ is the radiation dose measured at position $\mathrm{Z}$ along the scanner's main axis.

This equation is often used in a modified form

$$
\operatorname{CTDI}_{100}=\frac{1}{n T} \int_{-50 \mathrm{~mm}}^{50 \mathrm{~mm}} D_{a}(z) d z
$$

The dose absorbed in air $\mathrm{D}_{\mathrm{a}}(\mathrm{z})$ can be conveniently assessed with a $100-\mathrm{mm}$ standard pencil dose chamber. Typically, the dose distribution within the body cross section imparted by a CT scan is much more homogeneous than that imparted by radiography, but is still somewhat larger near the skin than in the body center. Therefore, a third measure, the weighted CTDI was introduced:

$$
C T D I_{w}=\frac{1}{3} C T D I_{100}^{\text {central }}+\frac{2}{3} C T D I_{100}^{\text {peripheral }}
$$

Similar measures with yet wider chambers are useful for CT systems with large numbers of detector rows [25].

\subsection{Lateral Dimension [26]}

The lateral (LAT) dimension is the side-to-side (left to right) dimension of the patient being scanned. This dimension can be assessed using electronic calipers on the standard PA CT radiograph that is acquired in most cases prior to a CT scan. In the absence of a $\mathrm{CT}$ radiograph, the lateral dimension could be determined using physical patient calipers, which are sometimes available in a radiology facility.

\subsection{AP Dimension}

Anterior-posterior dimension is the thickness of the body part of the patient being scanned anterior-posterior dimension, for example from the surface of the stomach to the surface of the back. To measure the AP dimension from a CT radiograph, a lateral projection would be necessary. The AP dimension can be easily measured using digital calipers on a conventional lateral $\mathrm{CT}$ radiograph, which is acquired prior to the CT scan under some circumstances. In the absence of a measurement from a CT radiograph, physical calipers can be used to make this measurement [26]. 


\subsection{AP + Lat Dimension}

This parameter is the sum of the lateral and AP dimensions. The analyses performed for this research will show that the sum of these two orthogonal dimensions is linearly related to the effective diameter.

\subsection{Effective Diameter [27]}

The effective diameter represents the diameter of the patient at a given location along the z-axis of the patient (in the cranial caudal dimension), assuming that the patient has a circular cross section. While some body parts approximate a circular section, many do not. The effective diameter can be thought of as the diameter of the circle whose area is the same as that of the patient cross section. To calculate this from the known AP and lateral dimensions, the patient is assumed to be elliptical in cross section, with the radii $r_{1}$ and $\mathrm{r}_{2}$ being:

$$
\begin{aligned}
& r_{1}=\frac{L A T}{2} \\
& r_{2}=\frac{A P}{2}
\end{aligned}
$$

The area, A, of the ellipse is computed using:

$$
A=\pi r_{1} r_{2}
$$

From the area of the patient's cross section, A, the effective diameter is computed as:

$$
\text { effectivediameter }=2 \sqrt{\frac{A}{\pi}}
$$

Combining Equations $4 \& 5$ through 6 , it can be seen that:

$$
\text { effectivediameter }=\sqrt{A P \times L A T}
$$

\subsection{Size Specific Dose Estimate (SSDE) [28]}

The Size specific dose estimate (SSDE) is defined as a patient dose estimate which takes into consideration corrections based on the size of the patient, using linear dimension measured on the patient or patient images. The SSDE values are specially based on the CTDIvol reported on CT scanner; however future modifications may include SSDE correction factors based on other patient phantom measurements. For example, CT measurement performed on different classes of CT phantoms and measurement protocols may provide the basis from which future SSDEs are built upon [29]. In all cases, the SSDE should correspond to tissue doses, not air kerma or other quantities. Thus, the f-factor (air kerma to tissue dose correction values, formerly known as Rontgen to rad conversion factors) should explicitly be a part of the SSDE metric.

The specific formula to estimate patient dose for a specific patient size is given by:

Size specific dose estimate $(\mathrm{SSDE})=\mathrm{f}_{\text {size }}{ }^{32} \mathrm{x} \mathrm{CTDI}^{32}$ vol

For the $32 \mathrm{~cm}$ diameter CTDIvol reference phantom, and
Size specific dose estimate $=\operatorname{SSDE}=\mathrm{f}_{\text {size }}{ }^{16} \mathrm{xCTDI}_{\mathrm{vol}}{ }^{16}$

For the $16 \mathrm{~cm}$ diameter CTDIvol reference phantom.

\subsection{Dose Length Product (DLP)}

Current computed tomographic (CT) scanners generate patient dose indexes of the volume CT dose index $\left(\mathrm{CTDI}_{\mathrm{vol}}\right)$ and the dose-length product (DLP) that are measured in 16and 32-cm-diameter acrylic phantoms [30-31]. $\mathrm{CTDI}_{\mathrm{vol}}$ is an index that quantifies the relative intensity of the radiation that is incident on the patient [32]. CT scanners that have patient size-specific scanning protocols would likely select lower $\mathrm{CTDI}_{\mathrm{vol}}$ values for pediatric patients but higher ones for oversized patients. The total amount of radiation delivered to the patient at a given examination, however, is also dependent on the CT scan length. The product of $\mathrm{CTDI}_{\mathrm{vol}}$ and scan length is the DLP, which can be used to quantify the total amount of radiation patients receive during a given scan [33]. The DLP is directly related to the patient (stochastic) risk and may be used to set reference values for a given type of CT examination to help ensure patient doses at CT are as low as reasonably achievable [34].

Mathematically DLP is defined as

$$
\mathrm{DLP}=\mathrm{CTDI}_{\mathrm{vol}} \times \text { scan length }
$$

Where,

scan length $=$ No of slice $\mathrm{x}$ slice thickness

\subsection{Effective Dose}

It is the central quantity for dose limitation in radiological protection in the international system of radiological protection devised by the International Commission on Radiological Protection (ICRP). The effective dose is not intended as a measure of deterministic health effect, which is the severity of acute tissue damage that is certain to happen [35].

Mathematically Effective dose is defined as

Effective dose $(\mathrm{E})=\mathrm{DLP} \times$ Tissue coefficient constant $(\mathrm{K})$

\subsection{CT Dosimetry Software}

Commercially available CT dosimetry software was used to compute patient ED and DLP. A CT dosimetry spreadsheet (ImPACT CT Patient Dosimetry Calculator, version0.99x; ImPACT, London, England) was used for all examinations reported in this study.

\subsection{Principles of Operation Components of a CT System}

A CT system consists of an x-ray subsystem, a gantry, a patient table, and a controlling computer. A high-voltage xray generator supplies electric power to the $\mathrm{x}$-ray tube, which usually has a rotating anode and is capable of withstanding the high heat loads generated during rapid multiple-slice acquisition. The gantry houses the x-ray tube, 
X-ray generator, detector system, collimators, and rotational frame. Most solid-state detectors are made of ceramic materials that produce light when exposed to ionizing radiation. Silicon photodiodes convert this light into an electrical signal. Collimators located near the x-ray tube and, on some systems, next to the detector are aligned so that scatter radiation is minimized and the x-ray beam is properly defined for scanning. The patient table can be moved both vertically and horizontally to accommodate various scanning positions. During a CT scan, the table moves the patient into the gantry and the x-ray tube rotates around the patient. As x-rays pass through the patient to the detectors, the computer acquires and processes data to form an image. The computer also controls the x-ray production, gantry motions, table motions, and image display and storage.

A CT scanner is made up of 4 main components, the gantry (frame) houses the X-ray source and detectors and has a large opening in the middle (patient port), the subject table which moves in and out of the patient port, the x-ray source and detectors, and a computer system that gathers information from the detectors and produces an image from the information [36].

\section{Results}

In order to measure effective dose using head protocol of a patient during CT scan, 25 patients ( 9 female and 16 male) were taken under our study. To optimize CT protocols, it is important to have a basic understanding of CT scan parameters, and their effects on image quality and radiation dose is required. CT protocols should be strictly optimized on the basis of body size, body region, clinical indication, and the availability of prior imaging. Before being start the works, we had been calibrated CT protocol with $32 \mathrm{~cm}$ diameter PMMA phantom. At the time of CT scan of head, a patient was laid on a patient table couch and exposed radiation for 2-3 seconds. Then using controlling computer command, 15-16 images of patient's brain were observed. These images were analyzed to mark the injured parts of brain. The images were divided into slices. From these different slices, AP and LAT dimensions were measured. For taking the image of a brain, a sufficient current (220 mAs), voltage $(120 \mathrm{Kv})$ were provided on $\mathrm{CT}$ scanner. The measured value of $\mathrm{CTDI}_{\mathrm{vol}}$ was $36.7 \mathrm{mGy}$. The LAT and AP were the range of (12.67-14.48) $\mathrm{cm}$ and (12.19-19.86) $\mathrm{cm}$ respectively [Table 1].The effective diameter was the range of (12.99-16.67) $\mathrm{cm}$. To calculate DLP, we had been used conversion factor [37], tissue coefficient constant [38], scan length. The DLP, SSDE and effective dose were the range of (165.15-183.50) mGy. Cm, and (73.76-85.14) mGy and (346587) $\mathrm{mSv}$ respectively [Table 2].To calculate effective dose, and tissue coefficient constant $(\mathrm{k})$ were used which shown in Table 2 [38]. The effective dose had been calculated with the help of equation (12).The effective doses were calculated from experimental data after CT scan of head or brain of patients.

Table 1. LAT and AP measurements of the Patients.

\begin{tabular}{|c|c|c|c|c|c|c|c|c|c|c|c|}
\hline $\begin{array}{l}\text { Patient } \\
\text { IDNo }\end{array}$ & sex & Age(years) & Region & $\begin{array}{l}\text { Scan } \\
\text { Time(sec) }\end{array}$ & $\begin{array}{l}\text { No. of } \\
\text { Slice }\end{array}$ & $\begin{array}{l}\text { Slice Length / } \\
\text { FOV (mm) }\end{array}$ & $\mathrm{CTDI}_{\mathrm{vol}}(\mathrm{mGy})$ & $\begin{array}{l}\text { Scan organ } \\
\text { mode/position }\end{array}$ & Angle & LAT(cm) & $\mathbf{A P}(\mathbf{c m})$ \\
\hline & & & & & & & 36.7 & Lateral & -6 & 13.1 & 12.9 \\
\hline 001 & $\mathrm{M}$ & 55 & Head & 20 & 10 & 210 & 36.7 & Lateral & -6 & 13.1 & 12.9 \\
\hline 002 & $\mathrm{M}$ & 40 & Head & 18 & 9 & 210 & 36.7 & Lateral & -3 & 12.85 & 16.18 \\
\hline 003 & $\mathrm{~F}$ & 55 & Head & 20 & 10 & 210 & 36.7 & Lateral & -6 & 13.18 & 17.27 \\
\hline 004 & $\mathrm{M}$ & 55 & Head & 18 & 9 & 210 & 36.7 & Lateral & -6 & 13.86 & 17.47 \\
\hline 005 & $\mathrm{M}$ & 60 & Head & 18 & 9 & 210 & 36.7 & Lateral & -3 & 14.48 & 19.13 \\
\hline 006 & $\mathrm{M}$ & 8 & Head & 20 & 10 & 210 & 36.7 & Lateral & -6 & 13.66 & 17.26 \\
\hline 007 & $\mathrm{M}$ & 80 & Head & 20 & 10 & 210 & 36.7 & Lateral & -3 & 13.76 & 17.54 \\
\hline 008 & $\mathrm{~F}$ & 50 & Head & 18 & 9 & 210 & 36.7 & Lateral & -3 & 13.43 & 16.33 \\
\hline 009 & $\mathrm{M}$ & 13 & Head & 18 & 9 & 210 & 36.7 & Lateral & -6 & 13.54 & 17.13 \\
\hline 010 & $\mathrm{M}$ & 38 & Head & 18 & 9 & 210 & 36.7 & Lateral & -6 & 14.21 & 17.66 \\
\hline 011 & $\mathrm{M}$ & 30 & Head & 18 & 9 & 210 & 36.7 & Lateral & -6 & 13.31 & 16.62 \\
\hline 012 & $\mathrm{M}$ & 60 & Head & 20 & 10 & 210 & 36.7 & Lateral & -6 & 13.4 & 15.37 \\
\hline 013 & $\mathrm{M}$ & 70 & Head & 20 & 10 & 210 & 36.7 & Lateral & -3 & 13.57 & 17.21 \\
\hline 014 & $\mathrm{~F}$ & 40 & Head & 18 & 9 & 210 & 36.7 & Lateral & -3 & 14.05 & 19.86 \\
\hline 015 & $\mathrm{~F}$ & 9 & Head & 20 & 10 & 210 & 36.7 & Lateral & -3 & 12.67 & 15.27 \\
\hline 016 & $\mathrm{~F}$ & 30 & Head & 20 & 10 & 210 & 36.7 & Lateral & -3 & 12.99 & 15.69 \\
\hline 017 & $\mathrm{~F}$ & 60 & Head & 20 & 10 & 210 & 36.7 & Lateral & -6 & 13.14 & 16.97 \\
\hline 018 & M & 70 & Head & 18 & 9 & 210 & 36.7 & Lateral & -3 & 13.58 & 17.12 \\
\hline 019 & $\mathrm{M}$ & 10 & Head & 20 & 10 & 210 & 36.7 & Lateral & -6 & 14.05 & 16.94 \\
\hline 020 & $\mathrm{~F}$ & 8 & Head & 20 & 10 & 210 & 36.7 & Lateral & -3 & 13.76 & 17.26 \\
\hline 021 & M & 40 & Head & 18 & 9 & 210 & 36.7 & Lateral & -6 & 13.38 & 17.28 \\
\hline 022 & $\mathrm{~F}$ & 45 & Head & 18 & 9 & 210 & 36.7 & Lateral & -3 & 12.98 & 16.38 \\
\hline 023 & $\mathrm{~F}$ & 5 & Head & 20 & 10 & 210 & 36.7 & Lateral & -6 & 13.46 & 16.98 \\
\hline 024 & M & 60 & Head & 18 & 9 & 210 & 36.7 & Lateral & -3 & 13.1 & 17.26 \\
\hline 025 & $\mathrm{M}$ & 65 & Head & 20 & 10 & 210 & 36.7 & Lateral & -3 & 13.24 & 17.53 \\
\hline
\end{tabular}


Table 2. Size specific effective dose estimation parameters.

\begin{tabular}{lllllllll}
\hline Patient ID & LAT+AP & Effective & ${ }^{1}$ Conversion & ${ }^{2}$ TissueCoefficientConstant & ScanLength & DLP & SSDE & EffectiveDose \\
\hline No. & Dia $(\mathbf{c m})$ & Dia $(\mathbf{c m})$ & Factor & $\left.\mathbf{( m S v G y}^{-1} \mathbf{c m}^{-1}\right)$ & $(\mathbf{c m})$ & $(\mathbf{m G y}-\mathbf{c m})$ & $(\mathbf{m G y})$ & $(\mathbf{m S v})$ \\
\hline 001 & 26.00 & 12.99 & 2.32 & 0.0021 & 5.0 & 183.50 & 85.14 & 385 \\
002 & 29.03 & 14.41 & 2.24 & 0.0021 & 4.5 & 165.15 & 82.20 & 346 \\
003 & 30.45 & 15.08 & 2.16 & 0.0021 & 5.0 & 183.50 & 79.27 & 385 \\
004 & 31.33 & 15.56 & 2.08 & 0.0021 & 4.5 & 165.15 & 76.33 & 346 \\
005 & 33.61 & 16.64 & 2.01 & 0.0021 & 4.5 & 165.15 & 73.76 & 346 \\
006 & 30.92 & 15.15 & 2.16 & 0.0032 & 5.0 & 183.50 & 79.27 & 587 \\
007 & 31.30 & 15.53 & 2.08 & 0.0021 & 4.5 & 165.15 & 76.33 & 346 \\
008 & 29.76 & 14.80 & 2.16 & 0.0021 & 4.5 & 165.15 & 79.27 & 346 \\
009 & 30.67 & 15.22 & 2.16 & 0.0021 & 5.0 & 183.50 & 79.27 & 385 \\
010 & 31.87 & 15.04 & 2.08 & 0.0021 & 5.0 & 183.50 & 76.33 & 385 \\
011 & 29.93 & 14.07 & 2.16 & 0.0021 & 5.0 & 183.50 & 79.27 & 385 \\
012 & 28.77 & 14.15 & 2.24 & 0.0021 & 4.5 & 165.15 & 82.20 & 346 \\
013 & 30.78 & 15.28 & 2.16 & 0.0021 & 4.5 & 165.15 & 79.27 & 346 \\
014 & 33.91 & 16.40 & 2.01 & 0.0021 & 5.0 & 183.50 & 73.76 & 385 \\
015 & 27.94 & 13.40 & 2.24 & 0.0032 & 4.5 & 165.15 & 82.20 & 528 \\
016 & 28.68 & 14.27 & 2.24 & 0.0021 & 5.0 & 183.50 & 82.20 & 385 \\
017 & 30.11 & 14.93 & 2.16 & 0.0021 & 4.5 & 165.15 & 79.27 & 346 \\
018 & 30.70 & 15.24 & 2.16 & 0.0021 & 4.5 & 165.15 & 79.27 & 346 \\
019 & 30.99 & 15.42 & 2.16 & 0.0021 & 5.0 & 183.50 & 79.27 & 385 \\
020 & 31.02 & 15.41 & 2.08 & 0.0032 & 5.0 & 183.50 & 76.33 & 587 \\
021 & 30.66 & 15.20 & 2.16 & 0.0021 & 4.5 & 165.15 & 79.27 & 346 \\
022 & 29.36 & 16.67 & 2.16 & 0.0021 & 4.5 & 165.15 & 79.27 & 346 \\
023 & 30.44 & 15.11 & 2.16 & 0.0040 & 5.0 & 183.50 & 79.27 & 734 \\
024 & 30.36 & 15.03 & 2.16 & 0.0021 & 5.0 & 183.50 & 79.27 & 385 \\
025 & 30.77 & 15.23 & 2.16 & 0.0021 & 4.5 & 165.15 & 79.27 & 346 \\
\hline
\end{tabular}

\section{Discussion}

Figure 1 describes the effective diameter of the head as a function of the AP dimension of the patient, over a large range of patient sizes. The best-fit curve using a second order polynomial across all data points demonstrates an excellent correlation coefficient of $\mathrm{R}^{2}=0.895$. In this case, for these data, the slope of the curve slightly increases as a function of AP dimension, resulting in a slight upward curvature in the plot.

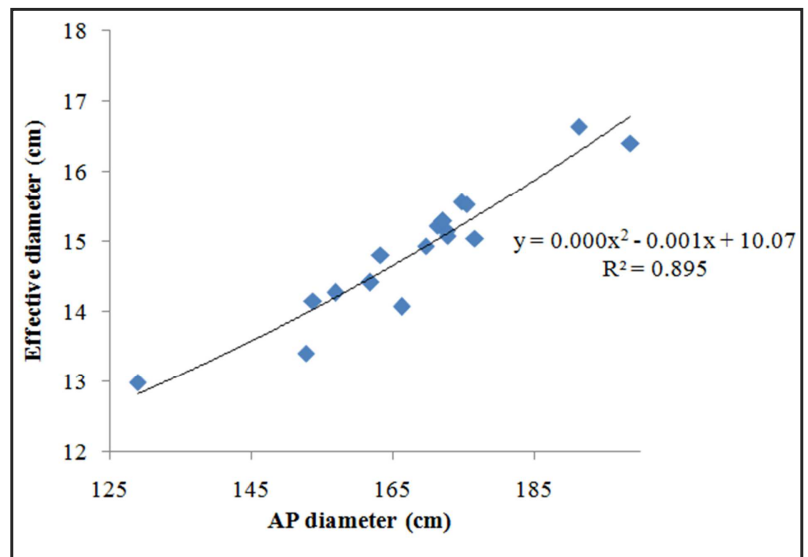

Fig. 1. The effective diameter as a function of AP dimension is illustrated. A slight upward curvature is seen in this relationship.

Figure 2 illustrates the effective diameter of the head as a function of the lateral dimension of the patient, over a large range of patient sizes. The best-fit curve using a second order polynomial across all data points demonstrates an excellent correlation coefficient of $\mathrm{R}^{2}=0.580$.In this case, however, the slope of the curve slightly decreases as a function of lateral dimension, resulting in a slight downward curvature in the plot.

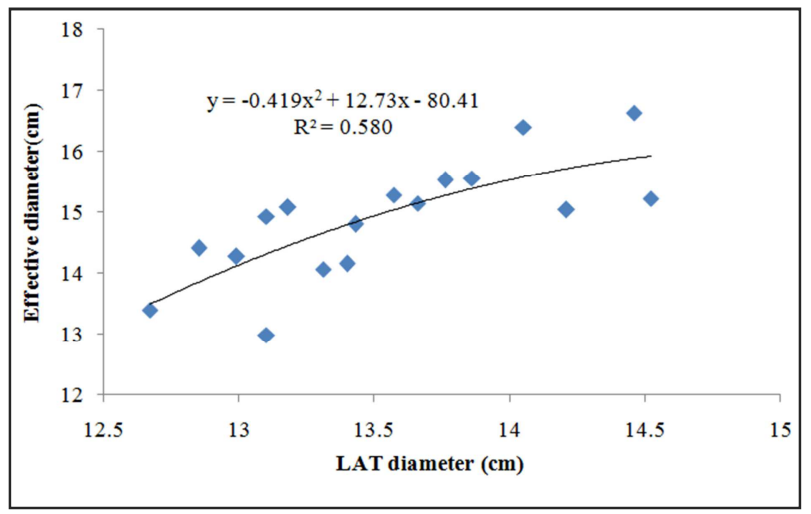

Fig. 2. The effective diameter as a function of lateral dimension is illustrated. A slight downward curvature is seen in this relationship.

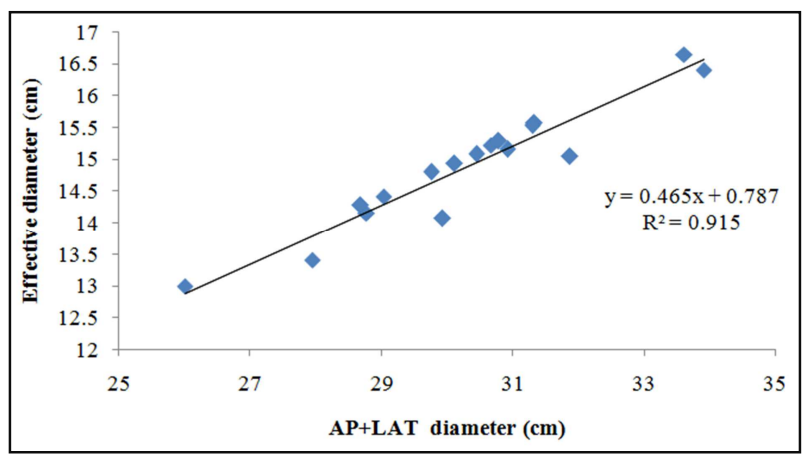

Fig. 3. The effective diameter is shown as a function of the sum of the anterior posterior and lateral dimensions of the patient. The upward and downward curvatures illustrated in figures 1 and 2 apparently compensate for each other, resulting in the linear relationship seen here. 
In the event that both the AP and lateral dimensions of the patient are known, the sum of these two dimensions can be used to estimate effective diameter. This relationship is illustrated in Figure 3 using data discussed previously, a liner relationship is observed with an excellent coefficient of 0.915. It is interesting to note that this parameter demonstrates a decidedly linear relationship with the effective diameter. Thus, the downward curvature associated with the lateral dimension (Figure 2) combines with the upward curvature associated with the AP dimension (Figure 1) to produce this linear relationship [28].

When the patient dimensions are not known, either from the cross-sectional CT images or a CT radiograph, the patient age may be known. Figure 4 provides the relationship between effective diameter and ages of the patient in our study. From this figure we calculated effective diameter corresponding to ages of the patient. While age may be used as an approximation, dose estimates based on patient size are considered more accurate and should be used when size information is available. From this plot, the effective diameter slightly increases with patient age that is comparable in ICRU 74.

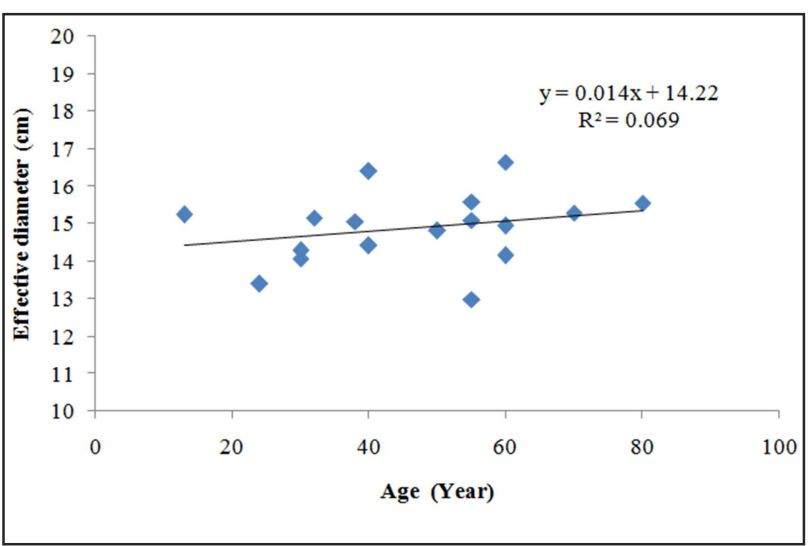

Fig. 4. The effective diameter (in cm) is shown as a function of Age (years).

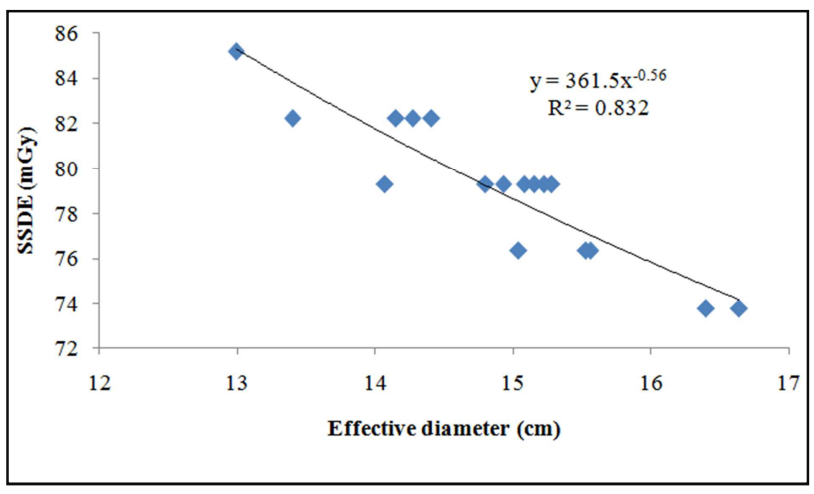

Fig. 5. The effective diameter is shown as a function of $\operatorname{SSDE}(m G y)$.

Figure 5 shows that the relationship between the size specific dose estimate (SSDE) and effective diameter. The best-fit curve using a second order polynomial across all data points demonstrates an excellent correlation coefficient $\mathrm{ofR}^{2}=0.832$. The SSDE decreases with effective diameter. For the effective diameter of the slice of patient's CT image, a doctor can easily evaluate the size-specific effective dose for CT scan of the patients. Without effective diameter a doctor or medical physicists can also calculate the sizespecific effective dose by knowing the patient's age.

\section{Conclusions}

The effective doses were evaluated from experimental data after CT scan of head or brain of patients. The estimated value of effective dose was in the range of (346-587) $\mathrm{mSv}$. It was also analyzed the relationship among effective diameter of a patient, AP, LAT, AP+LAT dimension, SSDE and age of the patients. The slope of the curve slightly increases as a function of AP dimension with effective diameter, resulting in a slight upward curvature in the plot. On the other hand, the slope of the curve slightly decreases as a function of lateral dimension, resulting in a slight downward curvature in the plot. For AP+LAT dimension and effective diameter of a patient, showed resulting in the linear relationship. By knowing the effective diameter of the slice of patient's CT image, a doctor can easily estimate the size-specific effective dose for CT scan of the patients. It is also noted that without knowing effective diameter a doctor or medical physicist can estimate the size-specific effective dose depend on the patient's age.

\section{Acknowledgments}

The author would like to thank Acota Clinic, Rajshahi, Bangladesh, for providing data supply.

\section{References}

[1] Mettler FA, Wiest PW, Locken JA et al (2000) CT scanning: patterns of use and dose. J Radiat Prot20(4):353-359.

[2] Bogdanich W (2009) Radiation overdoses point op dangers of CT scans. The New York Times; 15, October. Available from: http://www.nytimes.com/2009/10/16/us/16radiation.html.

[3] U.S. Food and Drug Administration (2010) White Paper: Initiative to reduce unnecessary radiation exposure from medical imaging. Silver Springs, MD; 2010. Available from:http://www.fda.gov/radiationemittingproducts/radiationsafety/radiationdosereduction/ucm1 99904.htm.

[4] Brenner DJ, Hall EJ (2007) Computed tomography - an increasing source of radiation exposure. N Engl J Med 357(22):2277-2284.

[5] NCRP (2009) Ionizing radiation exposure of the population of the United States. NCRP report no.160. Bethesda (MD): National Council on Radiation Protection and Measurements.

[6] Patterson A, Frush DP, Donnelly LF et al (2001) Helical CT of the body: are settings adjusted for pediatric patients? AJR 176(2):297-301.

[7] Donnelly LF, Emery A, Brody AS, et al. (2001) Minimizing radiation dose for pediatric body applications of single detector helical CT: strategies at a large Children's Hospital. AJR 176(2):303-306. 
[8] Brenner DJ, Elliston CD, Hall EJ net al (2001) Estimated risk of radiation-induced fatal cancer from pediatric CT. AJR 176(2):289-296.

[9] Brenner DJ, Elliston CD (2004) Estimated radiation risks potentially associated with full-body CT screening. Radiol 232(3):735-738.

[10] NRC (1990) Health effects of exposure to low levels of ionizing radiation: BEIR V. Washington DC: National Academies Press.

[11] NRC (2005) Health effects of exposure to low levels of ionizing radiation: BEIR VII - Phase 2. Washington DC: National Academies Press.

[12] Mettler FAJr, Wiest PW, Locken JA et al Kelsey CA. (2000) CT scanning: patterns of use and dose.J Radial Prot 20(4):353-359.

[13] SlovisTL (2002) The ALARA concept in pediatric CT: myth or reality? Radiology 223:5-6.

[14] Berdon WE, Slovis TL (2002) Where we are since ALARA andthe series of articles on CT dosein children and risk of long-term cancers: what has changed? Pediatr Radio32:699.

[15] Linton OW, Mettler FA (2003) National conference on dose reduction in CT, with an emphasis on pediatric patients. AJR Am J Roentgenol181:321-329.

[16] Shope T, Gagne R, Johnson G (1981) A method for describing the doses delivered by transmission $\mathrm{x}$-ray computed tomography. Med Phys8:488-495.

[17] Dixon RL (2003) A new look at CT dose measurement: Beyond CTDI. Med Phys 30(6):1272-1280.

[18] Boone JM (2007) The trouble with $\mathrm{CTD}_{100}$. Med Phys 34(4):1364-1371.

[19] McCollough CH (2008) CT dose: how to measure, how to reduce. Health Phys 95(5):508-517.

[20] McNitt-Gray MF (2002) APM/RSNA Physics Tutorial for Residents: Topics in CT. Radio Graphics 22(6): 1541-1553.

[21] AAPM 2008 Report of AAPM Task Group 23 of the Diagnostic Imaging Council CT Committee: the measurement, reporting, and management of radiation dose in CT $A A P M$ Report No. 96 (City Park, MD: American Association of Physicist in Medicine).

[22] IEC (2002) Medical Electrical Equipment. Part 2-44: Particular requirements for the safety of $\mathrm{x}$-ray equipment for computed tomography. IEC publication No. 60601-2-44. Ed. 2.1, International Electrotechnical Commission (I EC) Central Office: Geneva, Switzerland.

[23] Hill B, Venning AJ, Baldock C (2005) A preliminary study of the novel application of normoxic polymer gel dosimeters for the measurement of CTDI on diagnostic x-ray CT scanners. Med.Phys32:1589-1597.

[24] Rothenberg LN, Pentlow KS (2000) CT dosimetry and radiation safety, Categorical Course in Diagnostic Radiology Phyics. CT and US Cross-sectional Imaging171-188.
[25] Geleijns J, Salvadó MA, Bruin PW et al (2009) Computed tomography dose assessment for a $160 \mathrm{~mm}$ wide, 320 detector row, cone beam CT scanner. PhysMed Biol54: 3141-3159.

[26] AAPM (2011) Size-specific dose estimates (SSDE) in pediatric and adult body CT examinations AAPM Report No 204 (City Park, MD: American Association of Physicist in Medicine) 3.

[27] AAPM (2011) Size-specific dose estimates (SSDE) in pediatric and adult body CT examinations AAPM Report No 204 (City Park, MD: American Association of Physicist in Medicine) 4 .

[28] AAPM (2011) Size-specific dose estimates (SSDE) in pediatric and adult body CT examinations AAPM Report No 204 (City Park, MD: American Association of Physicist in Medicine) 13-19.

[29] AAPM (2010) Report of AAPM Task Group 111: The future of CT dosimetry: comprehensive methodology for the evaluation of radiation dose in x-ray computed tomography AAPM Report No 111 (City Park, MD: American Association of Physicist in Medicine).

[30] Galanski M, Hidajat N, Maier W et al (2000) Radiation exposure in computed tomography. 4th ed. Hamburg, Germany: CTB Publications.

[31] McNitt-Gray MF (2002) AAPM/RSNA physics tutorial for residents: topics in CT-radiation dose in CT. RadioGraphics22:1541-1553.

[32] IEC (2003) International standard of 60601-2-44 Ed2 Amendment 1: medical electrical equipment, Part 2-44particular requirements for the safety of x-ray equipment for computed tomography. Geneva, Switzerland: International Electrotechnical Commission.

[33] ICRP (2000) Recommendations of the international commission on Radiological protection30:7-45.

[34] Shrimpton PC, Hillier MC, Lewis MA et al (2006) National survey of doses from CT in the UK: 2003. Br J Radiol79:968980.

[35] ICRP (2007) Recommendations of the international commission on Radiological protection. ICRP Publication 103, New York.

[36] Robb RA (1982) X-ray computed tomography: from basic principles to applications. Annual Review of Biophysics and Bioengineering 11:177-201.

[37] AAPM (2011) Size-specific dose estimates (SSDE) in pediatric and adult body CT examinations AAPM Report No 204 (City Park, MD: American Association of Physicist in Medicine) 10-11.

[38] AAPM (2008) Report of AAPM Task Group 23 of the Diagnostic Imaging Council CT Committee: the measurement, reporting, and management of radiation dose in CT AAPM Report No. 96 (City Park, MD: American Association of Physicist in Medicine) 13. 